\title{
Can Nuts Consumption Modulate Cardiovascular Diseases? Report of a Case and Review of Literature
}

\author{
Viola Vargova ${ }^{1, *}$, Viola Mechirova ${ }^{1}$, Jan Fedacko $^{1}$, Rafai Ryber ${ }^{1}$, Daniel Pella ${ }^{1}$, \\ Agnieska Wilczynska ${ }^{2}$, Fabien De Meester ${ }^{2}$ and Ram B. Singh ${ }^{2}$ \\ ${ }^{I}$ Faculty of Medicine, PJ Safaric University, Kosice, Slovakia; \\ ${ }^{2}$ Tsim Tsoum Institute, Krakow, Poland
}

\begin{abstract}
Dietary benefits may be provided by haemostatic factors, omega-3 fatty acids, monounsaturated fatty acids (MUFA), vitamins, flavonoids, folic acids, plant sterols, alcohol and wine. Onion, garlic, spices, green tea and other herbs could be another dimention which may have a beneficial effects on human health and diseases. Nuts such as almonds, walnuts, pea nuts may have beneficial effects on cardiovascular risk factors. Epidemiological studies, suggested that nut eating was associated with diminished risk of coronary artery disease (CAD).
\end{abstract}

keywords: Nuts, cardiovascular diseases, MUFA, CAD.

\section{INTRODUCTION}

Diet appears to have a multidimentional beneficial effects on cardiovascular diseases; including atherogenesis, thrombosis, antioxidants, vitamins, antiinflammatory and lipid lowering effects [1-4]. Fruit, vegetable and nuts intake may have an independent role, separate from low fat calories and low density lipoprotein cholesterol (LDL-C). There is evidence that antioxidants protect the LDL cholesterol from oxidation in its atherogenic product oxidized LDL which is more attractive for macrophages during development of atherosclerosis [1,2]. Antioxidants also protect high density lipoprotein cholesterol (HDL) oxidation which is protective against atherothrombosis. Dietary dimentions may be provided by haemostatic factors, omega-3 fatty acids, monounsaturated fatty acids(MUFA), vitamins, flavonoids, folic acids, plant sterols, alcohol and wine [1-10]. Onion, garlic, spices, green tea and other herbs could be another dimention which may have a beneficial effects on human health and diseases $[3,4]$.

Nuts are concentrated sources of food energy and nutrients in a package built to carry health promotion and disease prehabilitation. Most physicians and dietitians avoid advising nuts to patients with obesity, diabetes and hypertension due to their high caloric value. However, epidemiological studies, suggested that nut eating was associated with diminished risk of coronary artery disease (CAD) [3-6]. In most studies on dietary patterns, nuts have been considered along with fruits, legumes and vegetables to demonstrate their influence on various risk factors of cardiovascular diseases (CVD) [7-18]. In most of these studies, w-3 content of dietary pattern has been reported to be inversely associated with ventricular premature contraction, acute myocardial infarction and inflammation [9-12]. Recent studies, indicate

*Address correspondence to this author at the Faculty of Medicine, PJ Safaric University, Kosice, Slovakia; Tel: 42155 6403409;

E-mails: viola_mechirova@yahoo.com,rbs@tsimtsoum.net that nuts consumption was inversely Associated with risk of hypertension in one study but no association in another [19, 20]. In the present work, we report a case in which increased consumption of walnut was associated with significant reduction in blood pressures.

\section{CASE REPORT}

An apparently healthy and asymptomatic male physician, aged 66 years, body weight $70.0 \mathrm{Kg}$ and waist circumference $96 \mathrm{~cm}$ had a history of having normal blood pressures during the last 20 years. In July third week, his blood pressure by mercury manometer was $130 / 92 \mathrm{mmHg}$ and pulse rate 82 per min after 5 min rest while sitting on a chair. His food consumption pattern based on 3 day record by dietary diary, was fruit, vegetable and nuts $410 \mathrm{~g} /$ day including almonds and walnuts $42 \mathrm{~g} / \mathrm{day}$, legumes and whole grains $350 \mathrm{~g} / \mathrm{day}$, refined carbohydrates $250 \mathrm{~g} /$ day, visible fat $35 \mathrm{~g} / \mathrm{day}$, milk $100 \mathrm{~g} /$ day. He was doing moderate exercise for about $20 \mathrm{~min}$ twice daily and pranayama breathing about $5 \mathrm{~min}$ twice daily. Alcohol intake was 4-10 drinks per week.

In the second week of December 2010, while coming after a visit to Poland and Moscow, he showed an increase in mean systolic and diastolic blood pressures. His blood pressure by mercury manometer was 142 systolic and $92 \mathrm{~mm} \mathrm{Hg}$ diastolic, pulse 80 per min and body weight $70 \mathrm{Kg}$ and waste circumference $97 \mathrm{~cm}$. During this trip to Europe, there was an increase in alcohol intake 10-12 drink per week but during recording of blood pressures, 6-8 drinks per week. Food consumption pattern showed lower intake of fruit, vegetable $300 \mathrm{~g} /$ day including nuts $20-25 \mathrm{~g} /$ day, legumes and whole grains $230 \mathrm{~g} /$ day, higher intake of refined carbohydrates $450 \mathrm{~g} / \mathrm{day}$ and visible fat intake $30 \mathrm{~g} / \mathrm{day}$, and milk $70 \mathrm{~g} / \mathrm{day}$.

In Jan 2010, he increased his consumption of walnuts (50-60g/day) while continuing exercise and pranayama regularly. After about 8 weeks, in March first week 2010, his blood pressure record by mercury manometer showed decrease in mean systolic and diastolic blood pressures but 
blood pressures were on the higher side of normal range. These changes in blood pressures were associated with body weight $70.3 \mathrm{Kg}$, waist circumference $96 \mathrm{~cm}$, blood pressure by mercury manometer $130 / 85 \mathrm{~mm} \mathrm{Hg}$, pulse rate $80 \mathrm{~min}$. On March 29, his blood pressures record showed $128 \mathrm{~mm}$ $\mathrm{Hg}$ systolic and $75 \mathrm{~mm} \mathrm{Hg}$ diastolic blood pressures recorded by mercury manometer as well as by automatic blood pressure instrument. His physical activity and pranayam breathing were continued twice daily without change, with increased consumption of fruits, vegetable (450g/day) including nuts $56 \mathrm{~g} /$ day, whole grains and legumes (350g/day), lower intake of refined carbohydrates $(150 \mathrm{~g} /$ day $)$. Visible fat intake (25-35g/day) and salt intake (5-6g/day) were similar during the last 6 months of the follow up. It is clear that one most significant change in the food consumption pattern was that the nuts intake was doubled and the increase was mainly in the walnuts. The consumption of alpha-linolenic acid which is rich in walnuts, whole grains, leafy vegetables and mustered oil increased from $1.2 \mathrm{~g} / \mathrm{day}$ to $2.6 \mathrm{~g} / \mathrm{day}$, as assessed by 3 days food intake records and with the help of nutrient composition of Indian foods tables.

\section{AMBULATORY BLOOD PRESSURE MONITORING}

Blood pressure by ambulatory blood pressure monitors (ABPM) were measured for 77 hours, half hourly, from December 26,2009 to December 28,2009. Mean (standard deviation,SD) systolic (154(22.47) $\mathrm{mmHg})$ and diastolic (87(14.42) $\mathrm{mmHg}$ ) blood pressures were higher. Mean pulse pressure was 88(11.08) BPM. Systolic/ diastolic limits during awake were $140 / 90 \mathrm{mmHg}$ and during sleep were 120/70mm Hg. Highest systolic blood pressure value was $237 \mathrm{~mm} \mathrm{Hg}$ and diastolic value $119 \mathrm{mmHg}$. Lowest systolic value was $95 \mathrm{~mm} \mathrm{Hg}$ and lowest diastolic value was $55 \mathrm{~mm}$ $\mathrm{Hg}$. After treatment with walnuts, blood pressures with ABPM were measured for 144 hours, from March 28,2010 to April 3,2010. Mean systolic blood pressure (129(13.95) $\mathrm{mm} \mathrm{Hg}$ ) and diastolic (77 (11.72) $\mathrm{mm} \mathrm{Hg}$ ) were substantially lower compared to earlier records taken in last December. Mean pulse pressure was 85 (9.4) BPM. Systolic/ diastolic limits were 140/90 during awakening and 120/70 during sleep. Highest systolic value was $171 \mathrm{~mm} \mathrm{Hg}$ and diastolic value $114 \mathrm{~mm} \mathrm{Hg}$. Lowest systolic value was $89 \mathrm{~mm} \mathrm{Hg}$ and diastolic value $51 \mathrm{~mm} \mathrm{Hg}$.

The decrease in mean blood pressures from December 2009 to April 2010 were, systolic $25 \mathrm{~mm} \mathrm{Hg}$ and diastolic $10 \mathrm{~mm} \mathrm{Hg}$, pulse pressure $3 \mathrm{~mm} \mathrm{Hg}$. There was a marked decrease in highest systolic value $66 \mathrm{~mm} \mathrm{Hg}$ as well as in highest diastolic value $5 \mathrm{~mm} \mathrm{Hg}$.

\section{COMMENTS AND REVIEW}

This case report indicates that increased consumption of nuts with increased content of ALA in the diet $(2.6 \mathrm{~g} / \mathrm{day})$ can decrease blood pressure without any reduction in body weight. There was no change in alcohol and salt intake. The finding suggest that a randomized, controlled trial in a large number of patients with a longterm follow up should be conducted to confirm these findings. Hypertension is a risk factor for CVD and dietary factors may play an important role in its prevention. The consumption of nuts could reduce the risk of hypertension, but scarce research has been done to evaluate this potential association $[19,20]$. To examine the association between nut consumption and incident hypertension, one prospective cohort of 15,966 participants from the Physicians' Health Study, who were free of hypertension at baseline were examined [19]. Nut consumption was assessed using a simple abbreviated food questionnaire and hypertension was self-reported. Cox regression to estimate relative risks of hypertension according to nut consumption was used. During 237,585 person-years of follow up, 8,423 new cases of hypertension occurred. Compared to subjects who did not consume nuts, multivariable adjusted hazard ratios $(95 \% \mathrm{CI})$ for hypertension were 0.97 (0.91-1.03), 0.98 (0.92$1.05), 0.96(0.89-1.03)$, and $0.82(0.71-0.94)$ for nut consumption of 1-2 times per month and 1, 2-6, and $>$ or $=7$ times/week, respectively. In a secondary analysis stratified by body mass index, there was an inverse relation between nut intake and hypertension in lean subjects ( $\mathrm{p}$ for trend 0.0019 ) but not in overweight or obese subjects ( $\mathrm{p}$ for interaction 0.0037). These data suggest that nut consumption is associated with a lower risk of hypertension in US male physicians and that such relation may be influenced by adiposity. However, this study did not report on association with other confounders like ALA and waist hip ratio.

Another recent study examined the association between nut consumption and the incidence of hypertension among 9919 Spanish university graduates followed-up biennially for a median of 4.3 years [20]. Food habits were assessed with a validated 136-item food-frequency questionnaire. Nut consumption was classified into four categories: rarely/never, 13/month, 1/week, and 2+/week. A participant was classified as an incident case of hypertension when, being free of hypertension at baseline, he/she subsequently reported a physician-made diagnosis of hypertension in at least one of the follow-up questionnaires. The incidence of hypertension was 12.4 per 1000 person-years. No association between nut consumption and incidence of hypertension was found, after adjusting for sex, age and other dietary and non-dietary potential confounders (hazard ratio [HR] for those in the highest vs. lowest nut consumption category $=0.77$ [IC 95\%: 0.46-1.30] $\mathrm{p}=0.795)$. Results were not modified when they awere stratified for analyses according to sex or to body mass index. These data do not provide evidence for an inverse association between nut consumption and incident hypertension in this cohort. Further results from cohorts and trials with a higher baseline risk of hypertension should be obtained to test this relationship. It seems that all nuts may not have sufficient beneficial effects on blood pressures.

In the Nurses health study [5], the association of nut consumption has been examined with risk of CAD, among 84 409 nurses during a follow up of 14 years. One out of 20 nurses consumed five or more servings of nuts a week whereas a third hardly consumed any nut ever. The risk of CAD after 14 years in the first group was half of that in the latter after adjustment of age and remained significantly lower by one third, even after adjustment for all available risk factors. A moderate risk was noted among subjects consuming moderate amount of nuts. A decreased risk from nut consumption was maintained across subgroups stratified to test for major confounding factors. The effect therefore appears large and significant, as high or higher than that in the statins trials. Dietary nut intake has been associated with a reduced risk of coronary artery disease (CAD) mortality, 
however the mechanism is unclear. Since components of nuts have antiarrhythmic properties, part of the benefit may be due to reduction in sudden cardiac death (SCD). In a prospective cohort study [3], increasing frequency of nut consumption at 12 months follow up, after a follow up of 17 years, was associated with a lower risk of SCD and other cardiovascular end points among 21,464 men participants enrolled in the US Physician,s Health Study, after controlling for known cardiac risk factors and other dietary habits ( $\mathrm{P}$ for trend 0.01). Compared with men who rarely or never consumed nuts, those who consumed nuts 2 or more times, per week had reduced risk of SCD (RR 0.53 ; 95\% CI 0.50-0.98). In contrast, nut intake was not associated with significant reduction in the risk of non sudden deaths or nonfatal myocardial infarction.

Cohort studies have limitations compared to power of randomized, controlled trials in determining causality, because there is always confounding by other factors. Frequent nut eaters differ in many respects from those who eat less or no nuts in the diet. In the nurses health study, of the four demarcated consumption groups, the high intake group came out most favourably for 16 of 23 factors, most of which appear to be independent rather than a consequence of nut consumption. The nurses health study and the US physicians health studies are postal studies whose participants have never been subjected to the clinical routines of other American cohort studies. Self reported hypertension was marginally least common among high consumption group, whereas hypercholesterolemia was marginally most common. The authors indicated eight constituents of nuts, that could be beneficial, but can not be more specific, which is a problem in demonstrating the independent effects of nuts in beneficial dietary patterns. However, randomized, controlled trials may provide better evidence regarding the role of nuts in the prevention of CVDs.

\section{CONTROLLED TRIALS OF NUT CONSUMPTION}

Epidemiologic and clinical trial evidence has demonstrated consistent benefits of nut and peanut consumption on CAD risk and associated risk factors [5-7, 18, 19]. These studies have considered various endpoints, including fatal $\mathrm{CAD}$, total CAD death, total CAD, and nonfatal myocardial infarct. A pooled analysis of 4 U.S. epidemiologic studies reviewed [18] showed that subjects in the highest intake group for nut consumption had an approximately $35 \%$ reduced risk of CAD incidence. The reduction in total CAD death was due primarily to a decrease in sudden cardiac death. Clinical studies have evaluated the effects of many different nuts and peanuts on lipids, lipoproteins, and various CAD risk factors, including oxidation, inflammation, and vascular reactivity $[18,19,21,22]$. Evidence from these studies consistently shows a beneficial effect on these CAD risk factors. The LDL cholesterol-lowering response of nut and peanut studies is greater than expected on the basis of blood cholesterol-lowering equations that are derived from changes in the fatty acid profile of the diet. Thus, in addition to a favorable fatty acid profile, nuts and peanuts contain other bioactive compounds that explain their multiple cardiovascular benefits. Other macronutrients include plant protein and fiber; micronutrients including potassium, calcium, magnesium, and tocopherols; and phytochemicals such as phytosterols, phenolic compounds, resveratrol, and arginine. Nuts and peanuts are food sources that are a composite of numerous cardioprotective nutrients and if routinely incorporated in a healthy diet, population risk of CAD would therefore be expected to decrease markedly.

Endothelial dysfunction is associated with CAD and its risk factors and is reversed by antioxidants and marine n-3 fatty acids. Walnuts are a rich source of both antioxidants and ALA, a plant n-3 fatty acid. It is possible that walnut intake may reverse endothelial dysfunction. In a randomized, crossover design, 21 hypercholesterolemic men and women were administered a cholesterol-lowering Mediterranean diet and a diet of similar energy and fat content in which walnuts replaced approximately $32 \%$ of the energy from monounsaturated fat [21]. Participants followed each diet for 4 weeks. After each intervention, we obtained fasting blood and performed ultrasound measurements of brachial artery vasomotor function. Eighteen subjects completing the protocol had suitable ultrasound studies. Compared with the Mediterranean diet, the walnut diet improved endotheliumdependent vasodilation and reduced levels of vascular cell adhesion molecule-1 ( $\mathrm{P}<0.05$ for both). Endotheliumindependent vasodilation and levels of intercellular adhesion molecule-1, C-reactive protein, homocysteine, and oxidation biomarkers were similar after each diet. The walnut diet significantly reduced total cholesterol $(-4.4+/-7.4 \%)$ and LDL cholesterol $(-6.4+/-10.0 \%) \quad(\mathrm{P}<0.05$ for both). Cholesterol reductions correlated with increases of both dietary alphalinolenic acid and LDL gamma-tocopherol content, and changes of endothelium-dependent vasodilation correlated with those of cholesterol-to-HDL ratios $(\mathrm{P}<0.05$ for all). It is likely that substituting walnuts for monounsaturated fat in a Mediterranean diet improves endothelium-dependent vasodilation in hypercholesterolemic subjects. This finding might explain the cardioprotective effect of nut intake beyond cholesterol lowering. Another study, was conducted to investigate whether the addition of walnuts or olive oil to a fatty meal have differential effects on postprandial vasoactivity, lipoproteins, markers of oxidation and endothelial activation, and plasma asymmetric dimethylarginine (ADMA) [22]. Compared with a Mediterranean diet, a walnut diet has been shown to improve endothelial function in hypercholesterolemic patients. In a randomized trial, in a crossover design, 12 healthy subjects and 12 patients with hypercholesterolemia were administered, 2 high-fat meal sequences to which $25 \mathrm{~g}$ olive oil or $40 \mathrm{~g}$ walnuts had been added [22]. Both test meals contained $80 \mathrm{~g}$ fat and $35 \%$ saturated fatty acids, and consumption of each meal was separated by 1 week. Venipunctures and ultrasound measurements of brachial artery endothelial function were performed after fasting and $4 \mathrm{~h}$ after test meals. In both study groups, flow-mediated dilation (FMD) was worse after the olive oil meal than after the walnut meal ( $p=0.006$, time-period interaction). Fasting, but not postprandial, triglyceride concentrations correlated inversely with FMD $(\mathrm{r}=-0.324 ; \mathrm{p}=0.024)$. Flowindependent dilation and plasma ADMA concentrations were unchanged, and the concentration of oxidized low-density lipoproteins decreased $(\mathrm{p}=0.051)$ after either meal. The plasma concentrations of soluble inflammatory cytokines and adhesion molecules decreased $(\mathrm{p}<0.01)$ independently 
of meal type, except for E-selectin, which decreased more $(p=0.033)$ after the walnut meal. It seems that adding walnuts to a high-fat meal acutely improves FMD independently of changes in oxidation, inflammation, or ADMA. Both walnuts and olive oil preserve the protective phenotype of endothelial cells. E-selectin is a proinflammatory factors which is responsible for rupture of the atherosclerotic plaque, resulting into atherothrombosis, indicating that walnut can prevent rupture of the hot plaque.Recent review on recent epidemiologic evidence on nut intake and health outcomes focuses on studies in which nut consumption is directly assessed or when nuts are included in a dietary score or pattern [23]. Epidemiologic studies have been remarkably consistent in showing an association between nut consumption and a reduced risk of CAD as described above. Some evidence has emerged recently to suggest health-protective benefits of nuts other than CAD. Frequent nut intake probably reduces risk of diabetes mellitus among women, but its effects on men are unknown. Evidence on the anticarcinogenic effects of nuts is somewhat limited because studies in the past 2 decades have examined only 3 tumor sites, and the benefits appear to be manifested only in women. However, the protective benefits of frequent nut consumption on gallstone diseases are observed in both sexes. Long-term nut consumption is linked with lower body weight and lower risk of obesity and weight gain. A dietary pattern or score that includes nuts is consistently related with beneficial health outcomes, and this provides an indirect evidence of the salutary benefits of nut consumption. More longitudinal studies are needed to clarify the possible effects of nuts on diseases other than CAD.

In brief, nuts are also rich in the favorable fatty acid profile containing high total and MUFA and w-3 fatty acids which probably contributes to the beneficial effects of nut consumption observed in epidemiologic studies related to $\mathrm{CAD}$ and diabetes and feeding trials. Besides fat, the complex matrices of nuts contain many bioactive compounds: vegetable protein, fiber, minerals, tocopherols, and phenolic compounds. By virtue of their unique composition, nuts are likely to benefit newer cardiovascular risk biomarkers, such as LDL oxidizability, soluble inflammatory molecules, and endothelial dysfunction. Protection of LDL oxidation by nut intake has been documented in some, but not all, clinical studies. Feeding one daily serving of mixed nuts may be associated with lower oxidized LDL concentrations. Regarding inflammation, cross-sectional studies have shown that nut consumption is associated with lower concentrations of circulating inflammatory molecules and higher plasma adiponectin, a potent antiinflammatory adipokine. Clinical studies with nuts have documented reduced inflammatory cytokine concentrations but no consistent changes of C-reactive protein. Only walnuts have been formally tested for effects on endothelial function [21,22]. After both walnut diets and single walnut meals, favorable vasoreactivity changes have been observed. Walnut consumption also reduces expression of endothelin 1, a potent endothelial activator, in an animal model of accelerated atherosclerosis. Beneficial effects on vascular reactivity may be ascribed to several constituents of walnuts: 1-arginine, the precursor of nitric oxide, alphalinolenic acid, and phenolic antioxidants. Although more studies are warranted, the emerging picture is that nut consumption beneficially influences cardiovascular risk beyond cholesterol lowering. Nutritional recommendations to prevent CVDs and promote longevity may need to focus on overall dietary patterns rather than individual food like nuts or a single nutrient $[13,16,17]$.

\section{WESTERN DIET}

Western diet is characterized with increased consumption of proinflammatory macronutrients such as w- 6 fatty acids, trans fatty acids(TFA) and saturated fatty acids(SFA) as well as refined carbohydrates which may produce hyperglycemia, oxidative stress, free fatty acids and proinflammatory substances [13-16]. Increased concentration of free fatty acids and proinflammatory cytokines are known to cause endothelial dysfunction whereas walnuts, fruits, vegetable improve endothelial functionm $[8,14,15,21,22]$. Glucose ingestion in normal subjects is associated with increased superoxide generation in leukocytes and mononuclear cells, as well as with raised amount and activity of nuclear factor- $\kappa \mathrm{B}$ (NF$\kappa \mathrm{B})$, a transcriptional factor regulating the activity of at least 125 genes, most of which are pro inflammatory [14, 15]. Increased consumption of refined carbohydrates also causes an increase in two other pro-inflammatory transcription factors, activating protein-1 (AP-1) and Egr-1, the first regulating the transcription of matrix metallo-proteinases and the second modulating the transcription of tissue factor and plasminogen activator inhibitor-1 $[14,15]$. It is possible that simultaneous supplementation with antioxidants such as coenzyme Q10 and w-3 fatty acids can modulate some of these mechanisms, resulting into decrease in these adverse effects. A mixed meal from a fast-food chain has also been shown to induce activation of NF- $\mathrm{KB}$ associated with the generation of reactive oxygen species (ROS) by mononuclear cells. Superoxide anion appear to be an activator of at least two major pro-inflammatory transcription factor, NF- $\kappa \mathrm{B}$ and AP1.These observations are consistent with previous findings, demonstrating that after oral or intravenous glucose challenges, in both normal subjects and patients with type 2 diabetes mellitus, there is an increased generation of ROS and raised circulating levels of proinflammatory cytokines, such as TNF- $\alpha$, IL-6 and IL-18 $[14,15]$. In apparently healthy subjects, a single high-fat meal produces endothelial activation, as evidenced by increased concentrations of the adhesion molecules VCAM-1 (vascular cell adhesion molecule-1) and ICAM-1 (intercellular adhesion molecule-1), in association with raised plasma concentrations of IL- 6 and TNF- $\alpha$. [7-9]. A high-fat meal $[14,15]$ may increase the circulating levels of IL-18, a pro-inflammatory cytokine supposed to be involved in plaque destabilization associated with the simultaneous decrease of circulating adiponectin, an adipocytederived protein with insulin sensitizing, anti-inflammatory, and antiatherogenic properties. These biochemical factors can also influence neuronal function and dysfunction that are majar aspects of Tsim Tsoum concept [17]. Further proof for the Tsim Tsoum concept has been provided in more recent studies on the effect of rapidly absorbed meals on risk factors of CVD and neuropsychiatric functions. (http://www. tsimtsoum.net/ editorials/tsimtsoum _editorial_2009-Kosice14th-WCCN-and-5th-ICCD.pdf). It seems that foods act like 
a nutraceutical. We develop an urge to eat those foods and nutrients which are inspired by our brain. In this mechanism taste is very important, which is observed in experiments with rats. Rats prefer choklates, although several foods were available to them in the cage. It is possible that food is an intoxication similar to alcohol, which we eat at the inspiration of our brain. Our brain allows us to eat only those foods, whose taste is already known to the brain. Consumption of a high-fat meal together with vegetable foods rich in natural antioxidants largely prevent the negative effects on endothelial function $[14,15]$. In particular, endothelial dysfunction acutely triggered by the consumption of a high-fat meal rich in saturated fatty acids is reduced by the simultaneous consumption of a vegetable serving including pepper $(100 \mathrm{~g})$ tomatoes $(100 \mathrm{~g})$, and carrots $(200 \mathrm{~g})$. It seems that these foods are slowly digested and absorbed without causing any significant increase in free radical stress and free fatty acids which is characteristic of Columbus foods.

\section{MEDITERRANEAN DIET}

Nuts are important component of Mediterranean diet. There is enormous epidemiological evidence that a Mediterranean type of diet may be protective against CVDs [16, 17]. In one study [16], a population of $>70000$ apparently healthy US women over the course of 18 years, was followed, assessing dietary intake repeatedly. By applying factor analysis, the authors identified 2 major dietary patterns. A greater adherence to the pattern labeled as prudent (characterized by a high consumption of plant foods such as vegetables, fruit, legumes, and whole grains as well as fish and poultry) was related to a $28 \%$ reduced risk of cardiovascular disease mortality and a $17 \%$ reduced risk of premature all-cause mortality. By contrast, a greater adherence to the pattern labeled as western (characterized by a high consumption of red and processed meat, refined grains, french fries, and sweets) was associated with a $22 \%$ increased risk of cardiovascular disease mortality, a $16 \%$ increased risk of cancer mortality, and a $21 \%$ increased risk of premature all-cause mortality. The observed associations were independent of known risk factors including age, smoking, physical inactivity, body mass index, and total caloric intake.

Western diet is proinflammatory and inflammation appears to be crucial in all stages of atherosclerosis, and few studies have investigated the effect of dietary fat on markers of inflammation related to this disease during the postprandial period [14-17, 24, 25]. To evaluate the chronic effects of dietary fat on the postprandial expression of proinflammatory genes in peripheral blood mononuclear cells (PBMCs) in healthy subjects, 20 healthy men followed three different diets for 4 weeks each, according to a randomized crossover design: Western diet: $15 \%$ protein, $47 \%$ carbohydrates (CHO), $38 \%$ fat (22\% saturated fatty acid (SFA)); Mediterranean diet: $15 \%$ protein, $47 \% \mathrm{CHO}, 38 \%$ fat $(24 \%$ monounsaturated fatty acid (MUFA)); CHO-rich and n-3 diet: $15 \%$ protein, $55 \% \mathrm{CHO},<30 \%$ fat $(8 \%$ polyunsaturated fatty acid (PUFA)). After 12-h fast, volunteers were given a breakfast with a fat composition similar to that consumed in each of the diets-butter breakfast: 35\% SFA; olive oil breakfast: 36\% MUFA; walnut breakfast: 16\% PUFA, 4\% alphalinolenic acid (LNA). The butter breakfast induced a higher increase in tumor necrosis factor (TNF)-alpha messenger RNA (mRNA) expression than the olive oil or walnut breakfasts $(\mathrm{P}=0.014)$ in PBMCs. Moreover, a higher postprandial response in the mRNA of interleukin (IL)-6 with the intake of butter and olive oil breakfasts was found than with the walnut breakfast $(\mathrm{P}=0.025)$ in these cells. However, the effects of the three fatty breakfasts on the plasma concentrations of these proinflammatory parameters showed no significant differences $(\mathrm{P}=\mathrm{N}$.S.). It seems that consumption of a butter-enriched meal elicits greater postprandial expression of proinflammatory cytokine mRNA in PBMCs, compared to the olive oil and walnut breakfasts. Longterm consumption of walnuts as breakfast may be associated with significant reduction in the proinflammatory cytokines in the tissues. Esposito et al. administered Mediterranean diet among patients of metabolic syndrome compared to standard diet [25]. There was a significant increase in endothelial function in the Mediterranean diet group compared to control subjects.

\section{THE INDIAN EXPERIMENT OF INFARCT SURVIVAL}

It has been suggested that a western diet where refined starches and sugars are rapidly absorbed, should be avoided in patients with acute coronary syndromes $[2,25-30]$ and administer diet which is beneficial to vascular endothelium and myocardium. There is limited evidence regarding the role of dietary intervention in patients with AMI [2, 26-30]. The aim of the Indian experiment was to determine the effects of a diet rich in w-3 fatty acids, vitamins, minerals and antioxidants (fruits, vegetables, legumes, walnuts, almonds, fish, mustered oil) and low in refined carbohydrates, in patients with (AMI, Tables 1-3). All patients with a diagnosis of ACS were assigned to an intervention $\operatorname{diet}(\mathrm{n}=204)$ or a control $\operatorname{diet}(n=202)$ within 48hours of the onset of the symptoms of AMI [26, 27].The intervention group was advised to consume $600 \mathrm{~g} /$ day of fruits, vegetables, legumes and almonds and walnuts, in a soup or semisolid form. Tomato soup, skim milk and curd (yogurt) were commonly used to mix crushed almonds and walnuts and other foods, which were grilled with mustered oil. The control group was advised a low fat diet consistent with National Cholesterol Education Program. (Tables 1, 2). Clinical characteristics, time elapsed from symptom onset to the index infarction, site of infarction, drug therapy and final diagnosis were comparable between the two groups. Intake of foods and selected nutrients was assessed during the 1 week and after 1 year. After 1 week, plasma lipid peroxides, vitamin $\mathrm{C}$ and lactate dehydrogenase levels were determined. Compared with the control groups, patients allocated to the dietary intervention consumed significantly greater amounts of fruit, vegetables, pulses, almonds, walnuts, oils and fish, both during the first trial week and 1 year after AMI $[26,27]$. The consumption of n-3 fatty acids was also significantly greater in the intervention group than in the control group $(1.8 \pm 0.66$ versus $0.65 \pm 0.4 \mathrm{~g}$ day $\left.{ }^{-1} \mathrm{P}<0.01\right)$. The consumption of proinflammatory foods, such as butter and clarified butter, refined starches and sugar were significantly greater in the control group than in the intervention group. Plasma lipid peroxide level decreased significantly in the intervention group compared with the control group, indicating a decrease in oxidative stress which is protective against proinflammatory IL-6 
Table 1. Effect of w-3 Fatty Acid Rich Diet in Patients with Acute Myocardial Infarction [26, 27] (Tubers not Included)

\begin{tabular}{|c|c|c|c|c|}
\hline Foods and Nutrients & 4-7 Days & After 1 Years & 4-7 Days & After 1 Years \\
\hline Fruits and vegetables (g. day-1) & $508.4 \pm 28.66^{* *}$ & $575 \pm 91.4^{* *}$ & $254.4 \pm 17.2$ & $220.5 \pm 19.6$ \\
\hline Legumes and pulses (g. day-1) & $80.5 \pm 6.6 * *$ & $95.0 \pm 8.9 * *$ & $52.5 \pm 4.6$ & $45.6 \pm 5.6$ \\
\hline Fish (g. day-1) & $52.5 \pm 6.5 * *$ & $22.4 \pm 4.1 * *$ & $20.2 \pm 3.1$ & $10.5 \pm 3.5$ \\
\hline Chicken (g. day-1) & - & $10.2 \pm 3.2 *$ & $76.2 \pm 6.5$ & $66.5 \pm 10.5$ \\
\hline Mustard or soybean oil & $18.4 \pm 3.9 *$ & $31.5 \pm 5.5 * *$ & $10.5 \pm 2.3$ & $6.8 \pm 2.8$ \\
\hline Butter or clarified butter (g. day-1) & $2.5 \pm 0.6 * *$ & $3.3 \pm 0.71 *$ & $10.5 \pm 2.6$ & $12.6 \pm 3.5$ \\
\hline Bread,biscuits (g. day-1) & $10.6 \pm 2.2 *$ & $25.5 \pm 6.2 * *$ & $230.6 \pm 20.1$ & $212.2 \pm 18.1$ \\
\hline Rice and wheat cereals (g. day-1) & $25.6 \pm 2.4$ & $30.6 \pm 5.5$ & $30.2 \pm 3.1$ & $35.6 \pm 4.8$ \\
\hline Honey or raisins (g. day-1) & $2.6 \pm 0.8$ & $5.5 \pm 1.2$ & - & - \\
\hline Sugar (g. day-1) & $16.4 \pm 3.7 *$ & $12.6 \pm 3.4^{*}$ & $25.5 \pm 5.4$ & $30.5 \pm 7.6$ \\
\hline Alpha-linolenic acid (g. day-1) & $1.0 \pm 0.46^{* *}$ & $1.8 \pm 0.66^{* *}$ & $0.5 \pm 0.11$ & $0.65 \pm 0.14$ \\
\hline Ascorbic acid (mg. day-1) & $474 \pm 70.5^{* *}$ & $440.6 \pm 100.6^{* *}$ & $150.2 \pm 10.6$ & $160 \pm 38.0$ \\
\hline
\end{tabular}

$\mathrm{P}$ values were obtained by comparison of intervention and control groups after 1 week and after 1 year. $*=\mathrm{P}<0.05$, $* * \mathrm{P}<0.01$.

Table 2. Effect of Dietary Intervention on Oxidative Stress, Vitamins and Cardiac Damage in Relation to Dietary Alpha-Linolenic Acid [26]

\begin{tabular}{|l|l|l|l|l|}
\hline & \multicolumn{2}{|l|}{ Intervention group (n=204) } & \multicolumn{2}{l|}{ Control group (n=202) } \\
\hline & Baseline, $\mathbf{3}^{\text {rd }}$ day & 7th day & Baseline, $\mathbf{3}^{\text {rd }}$ day & 7th day \\
\hline \hline Dietary alpha-linolenic acid(g/day) & $1.0 \pm 0.46$ & $1.8 \pm 0.66^{*}$ & $0.5 \pm 0.11$ & $0.65 \pm 0.4$ \\
\hline Plasma ascorbic acids $\left(23.2 \pm 3.2, \mu \mathrm{mol} .1^{-1}\right)$ & $7.38 \pm 1.7$ & $30.6 \pm 4.7^{* *}$ & $7.9 \pm 1.7$ & $15.8 \pm 3.8$ \\
\hline Plasma lipid peroxides $\left(1.4 \pm 0.41, \mathrm{pmol} .1^{-1}\right)$ & $3.56 \pm 0.71$ & $2.9 \pm 0.62^{*}$ & $3.48 \pm 0.7$ & $3.38 \pm 0.62$ \\
\hline Lactate dehydrogenase $\left(216.5 \pm 36.5, \mathrm{IU}^{-1} 1^{-1}\right)$ & $228.8 \pm 48.6$ & $656.5 \pm 75.6^{*}$ & $224.2 \pm 45.4$ & $785.4 \pm 81.2$ \\
\hline
\end{tabular}

$\mathrm{P}$ value was obtained by comparison of intervention and control groups on the seventh day after onset of symptoms of myocardial infarction. Values are mean $+\mathrm{SD}, *=\mathrm{P}<0.05$, $* *=\mathrm{P}<0.01$.

and TNF-alpha as well as endothelial dysfunction, although these data were not measured in our study. Lactate dehydrogenase (LDH) level increased less in the intervention group than in the control group, indicating that myocardial damage was prevented by the cardioprotective diet. The increased intake of n-3 fatty acids from mustard oil and walnuts associated with the Mediterranean diet might be responsible for the significant reduction in the cardiac enzyme LDH and lipid peroxides in the intervention compared with the control group. Total cardiac events, including fatal and non-fatal myocardial infarctions and sudden cardiac deaths, were significantly lower in the intervention group compared with the control group, both after 6 weeks [26] as well as after one year [27].

The effects of 1 year of treatment with fish oil (122 patients, eicosapentaenoic acid, EPA1.08g day ${ }^{-1}$ ) mustard oil (120 patients), alpha-linolenic acid $2.9 \mathrm{~g} \mathrm{day}^{-1}$ ), and no treatment (118 patients, placebo group) on the outcome of patients with suspected AMI were compared in a randomized, placebo-controlled trial) [28]. Treatment were administered within, on average 18 hour of onset of symptoms. Clinical characteristics, extent of cardiac damage and rise of cardiac enzymes and lipid peroxides were comparable among the 
Table 3. Effect of Cardioprotective Diet on Cardiac Events after one Year [26, 27]

\begin{tabular}{|c|c|c|}
\hline & Intervention Group (n=204) & Control Group (n=202) \\
\hline \hline Non-fatal myocardial infarction & $30(15)^{* *}$ & $48(24)$ \\
\hline Fatal myocardial infarction & $13(6)^{*}$ & $19(9)$ \\
\hline Sudden cardiac death(<1h) & $7(3)^{*}$ & $1(0.5)$ \\
\hline Suspected cardiac death & - & $2(1.0)$ \\
\hline Death due to stroke & $1(0.5)$ & $1(0.5)$ \\
\hline Death due to cancer & - & $34(17)$ \\
\hline Total cardiac mortality & $20(10)^{* *}$ & $38(19)$ \\
\hline Total mortality & $21(10)^{* *}$ & $86(43)$ \\
\hline Total cardiac events and deaths & $51(25)^{* *}$ & \\
\hline
\end{tabular}

$\mathrm{P}$ value was obtained by comparison of intervention and control group by a $\mathrm{Z}$ test for proportions. $\mathrm{P}<0.05$, $* *=\mathrm{P}<0.01$.

three groups at study entry. After randomization, angina pectoris (18.0 and 21.6 versus $42.3 \%$ ), arrhythmias (13.1 and 13.3 versus $28.7 \%)$ and poor left ventricular function $(22.8$ and 26.6 , versus $47.4 \%$ ) were significantly lower in the fish oil and mustard oil treatment groups compared with the placebo group. Sudden cardiac deaths (1.6 and 1.6 versus $6.6 \%$ ), total cardiac deaths (11.4 and 13.3 versus $22.0 \%$ ) non-fatal infarctions (13.0and 15.0, versus $25.4 \%$ ), and total cardiac events 24.5 and 28.2 versus $47.4 \%$ ) were also significantly lower in the two intervention groups. A modest improvement in dyslipidemia and a decrease in oxidative damage were observed in the fish oil and mustard oil groups but not in the placebo group. On the third and the fifth day after AMI, serum glutamic oxalo-transaminase (SGOT) and LDH cardiac enzymes showed greater decline in the fish oil and mustard oil groups compared with the placebo group. In a recent study [8], a total of 117 volunteers completed the 12week trial. Participants in the 1-, 3-, and 6-portions/d groups reported consuming on average $1.1,3.2$, and 5.6 portions of fruit and vegetables, respectively, and serum concentrations of lutein and $\beta$-cryptoxanthin increased across the groups in a dose-dependent manner. For each 1-portion increase in reported fruit and vegetable consumption, there was a $6.2 \%$ improvement in forearm blood flow responses to intraarterial administration of the endothelium-dependent vasodilator acetylcholine $(P=0.03)$. There was no association between increased fruit and vegetable consumption and vasodilator responses to sodium nitroprusside, an endotheliumindependent vasodilator.

\section{THE LYON DIET HEART STUDY}

The Lyon Diet Heart Study hypothesized that, because the lowest rates of cardiovascular diseases in the world were observed in populations either following a Mediterranean diet or a diet low in omega- 6 fatty acids but rich in omega- 3 fatty acids, the best strategy to reduce the rate of complications in patients with established CHD should be to adopt an omega-3 fatty acid-rich Mediterranean diet which is characterised with fruits, vegetables, nuts, poultry and olive oil. It is a secondary prevention trial designed to test the hypothesis that a Mediterranean ALA-rich diet may improve the prognosis of patients having survived a first acute myocardial infarction (AMI). A striking protective effect of the Mediterranean diet was reported with a 50-70\% reduction of the recurrence after 4 years of follow-up. Briefly, as regards lipids the experimental Mediterranean diet tested in the trial supplied less than $30 \%$ of energy from fats and less than $8 \%$ of energy from saturated fats. Regarding essential fatty acids, the intake of linoleic acid was restricted to $4 \%$ of energy and the intake of ALA made up more than $0.6 \%$ of energy. In practical terms, the dietary instructions were detailed and customized to each patient and can be summarized as: more bread, more cereals, more legumes and beans, more fresh vegetable, nuts and fruits, more fish, less meat (beef, lamb, pork) and delicatessen, which were to be replaced by poultry; no more butter and cream to be replaced by an experimental canola oil-based margarine. This margarine was chemically comparable with olive oil but slightly enriched in linoleic acid and mostly in ALA, the two essential fatty acids. Finally, the oils recommended for salad and food preparation were exclusively olive and canola oils. Two other major components of the traditional Mediterranean diet, in addition to a low omega-6/omega- 3 fatty acids ratio, are low saturated fat intake and high oleic acid intake. Patients also had to meet these two major criteria of a healthy diet. Thus, to meet the criteria of a Mediterranean diet, patients had to drastically reduce the consumption of foods rich in saturated (essentially animal) fat. Among vegetable oils, only olive oil (despite its lack of ALA) and canola oil (despite its very high amounts of linoleic acid) have a fatty acid composition in line with our strategy. Thus, the patients were advised to use both oils. Because of their high content in linoleic acid; soybean, sunflower and safflower oils should not be used daily for food preparation and salad dressing. Peanut oil is too rich in saturated fatty acids and linoleic acid, and linseed (flaxseed) oil is too rich in polyunsaturated fatty acids. In theory, the best option should be to vary the use of several oils.

To simplify, all subjects were advised and to the exclusive use of olive and canola oils. This exclusive use of olive 
and canola oils (and of canola-oil based margarine instead of butter to spread on the bread) to prepare meals and salad was a major issue in that trial as it resulted in significant differences in the fatty acid composition of both circulating plasma (essentially lipoproteins) and cell membrane phospholipids. The main differences between groups in platelet phospholipid fatty acids were not seen at the level of individual fatty acids (ALA is almost undetectable in cell membranes) but for the entire family of each group. Significant differences were also seen for the ratio of omega-6 to omega-3 fatty acids. Dietary approaches including fruts, vegetables, whole grains and nuts in conjunction with mustered oil or canola oil have been suggested by Renaud et al., Mechorova et al. and Singh et al. in several other clinical studies [31-34]. Singh et al. administered fruits, vegetables, legumes and nuts $(600 \mathrm{~g} /$ day $)$

To 310 patients and control diet to 315 patients with high risk of CAD [33, 34]. After a follow up of 12 weeks there was a significant reduction in systolic and diastolic blood pressures, blood glucose and blood lipoproteins, with an increase in HDL cholesterol. Addition of exercise to dietary changes, was associated with greater decrease in all these biomarkers and blood pressures.

\section{THE INDO-MEDITERRANEAN DIET STUDY}

Singh et al. [35] tested an 'Indo-Mediterranean diet' in 1000 patients in India, with existing coronary disease or at high risk for coronary disease. When compared with the control diet, the intervention diet characterized by increased intake of mustard or soyabean oil, nuts (walnuts, almonds), vegetables, fruits and whole grains-reduced the rate of fatal myocardial infarction by one-third and the rate of sudden death from cardiac causes by two-thirds. Recent clinical and epidemiological studies [9-12] indicate a significant association of w-3 fatty acid status and risk of ventricular premature beats, AMI and inflammation. The Indian experiments were the first to provide a scientific proof that dietary intervention characterized with fruits, vegetables, walnuts and almond in conjunction with mustered oil could be cardioprotective against MI and its complications; heart failure and arrhythmias, blood pressures [26-29] which was later confirmed in other more scientific studies [8, 9, 17-23, 36, 37].

Given the available evidence accumulated on plant omega-3 (w3-EFA) and by Others on animal omega-3 (w3HUFAs), it may be recommended that we extend on the evolutionary selected essentiality of plant omega-3 on heart rhythms and on the evolutionary selected 'conditionally' essentiality of animal omega-3 on a mesor blood pressure (Fabien De Meester, Personal communication, April 2010). From an evolutionary standpoint, plants precede animals and chronomes precede genomes. Animal clock genes are therefore mostly inherited from plants, which support the hypothesis that they must be regulated by plant omega-3 [37]. In a retrospective study of 111 hypertriglyceridemic subjects with untreated normal-high blood pressure that were prescribed 2 g/day PUFA supplementation for 12 months, results indicate decreases in systolic (mean decrease: $2.7 \mathrm{~mm}$ $\mathrm{Hg}$ ) and diastolic blood pressure (mean decrease: $1.3 \mathrm{~mm}$ $\mathrm{Hg}$ ) and basal heart rate (mean decrease: $4 \mathrm{bpm}$ ). Thus, the authors conclude, "In our retrospective study, highly purified omega-3 PUFA long-term supplementation is associated with a significant reduction in SBP, DBP, pulse pressure (PP), and basal heart rate in hypertriglyceridemic patients with normal-high blood pressure" [36].

In a randomized, crossover study involving 20 Chinese patients with type 2 diabetes and mild hyperlipidemia, results indicate that eating almonds may improve adiposity, glycemic control, and lipid profile [38]. After a two-week run-in period, patients were assigned to either a control $\mathrm{Na}$ tional Cholesterol Education Program step II diet (control diet) or an almond diet (replace $20 \%$ of total daily calorie intake; approx. $60 \mathrm{~g} /$ day) for 4 weeks. After a 2 week washout period the interventions were crossed over for another 4 weeks. Almond diet was associated with significant decreases in body fat, total cholesterol, low-density lipoprotein cholesterol, the ratio of low-density lipoprotein cholesterol to high-density lipoprotein cholesterol, plasma apolipoprotein (apo) B levels, apo B/apo A-1 ratio, nonesterified fatty acid, fasting insulin, fasting glucose, and homeostasis model assessment of insulin resistance index, compared with control diet. Thus, the authors of this study co nclude, "Our results suggested that incorporation of almonds into a healthy diet has beneficial effects on adiposity, glycemic control, and the lipid profile, thereby potentially decreasing the risk for cardiovascular disease in patients with type 2 diabetes mellitus.

\section{ACKNOWLEDGEMENTS}

are due to the International College of Nutrition, International College of Cardiology, Kosice, Slovakia, and The Tsim Tsoum Institute for support to write this review.

\section{REFERENCES}

[1] Fehily AM, Pickering JE, Yarnell JW, Elwood PC. Dietary indices of atherogenicity and thrombogenicity and ischaemic heart disease risk: the Caerphilly prospective study. Br J Nutr 1994; 71: 240-57.

[2] Singh RB, Fedacko J, Pella D, De Meester F, Moshiri M, Aroussy EW. Super foods dietary approaches for acute myocardial infarction. World Heart J 2010; 2: 1-8.

[3] Albert CM, Gaziano JM, Willet WC, Mension JE. Nut consumption and decreased risk of sudden cardiac death in the Physicians Health Study. Arch Intern Med 2002; 162: 1582-7.

[4] Tunstall-Pedoe H. Nuts to you: Eating nuts though it is unclear why. BMJ 1998; 317: 132-3.

[5] Hu FB, Stampfer JF, Manson JE, et al. Frequent nut consumption and risk of coronary heart disease in women: prospective cohort study. BMJ 1998; 317: 1341-5.

[6] Frager GE, Sabate J, Beeson WL, Strahan TM. A possible protective effect of nut consumption on risk of coronary heart disease: The Adventist Health Study. Arch Intern Med 1992; 152: 1410-24.

[7] Gramenzi A, Gentile A, Fasoli M, Negri E, Parazzini F.La Vecchia C. Association between certain foods and risk of acute myocardial infarction in women. BMJ 1990; 300: 771-3.

[8] McCall DO, McGartland CP, McKinley MC, et al. Dietary intake of fruits and vegetables improves microvascular function in hypertensive Subjects in a Dose-Dependent Manner. Circulation 2009; 119: 2153-60.

[9] Smith PJ, Blumenthal JA, Babyak MA, et al. Association between n-3 fatty acid consumption and ventricular ectopy after myocardial infarction. Am J Clin Nutr 2009; 89: 1315-20.

[10] Campos H, Baylin A, Willett WC. Alpha-Linolenic acid and risk of nonfatal acute myocardial infarction. Circulation 2008; 118: 33945.

[11] Harris WS, Reid KJ, Sands SA, et al. Blood omega-3 and trans fatty acids in middle aged acute coronary syndrome patients. Am J Cardiol 2007; 99: 154-8.

[12] Micallef MA, Munro IA, and Garg ML. An inverse relationship between plasma $\mathrm{n}-3$ fatty acids and C-reactive protein in healthy individuals. Eur J Clin Nutr 2009; 63(9): 1154-6. 
[13] Fung TT, Malik V, Rexroad KM, Manson JE, Willett WC, Hu FB. Sweetened beverage consumption and risk of coronary heart in women. Am J Clin Nutr 2009; 89: 1037-42.

[14] Esposito K, Glugliano D. Diet and inflammation:a link to metabolic and cardiovascular diseases. Eur Heart J 2006; 27: 15-20.

[15] Vogel RA. Eating, vascular biology, and atherosclerosis: a lot to chew on. Eur Heart J 2006; 27: 13-4.

[16] Heidemann C, Schulze MB, Franco OH, van Dam RM, Mantzoros CS, Hu FB. Dietary patterns and risk of mortality from cardiovascular disease, cancer, and all causes in a prospective cohort of women. Circulation 2008; 118: 230-7.

[17] Singh RB, De Meester F, Pella D, Watson RR, Basu TK. Globalization of dietary wild foods protect against cardiovascular disease: A scientific statement from the International College of Cardiology, Columbus Paradigm Institute and International College of Nutrition. Open Nutra J 2009; 2: 42-5.

[18] Ros E. Nuts and novel biomarkers of cardiovascular disease. Am J Clin Nutr 2009; 89: 1649S-56S.

[19] Djousse L, Rudich T, Gaziano JM. Nut consumption and risk of hypertension in US male physicians. Clin Nutr 2009; 28: 10-4.

[20] Martínez-Lapiscina EH, Pimenta AM, Beunza JJ, Bes-Rastrollo M, Martínez JA, Martínez-González MA. Nut consumption and incidence of hypertension: The SUN prospective cohort. Nutr Metab Cardiovasc Dis 2009; 20(5): 359-65.

[21] Ros E, Núñez I, Pérez-Heras A, et al. A walnut diet improves endothelial function in hypercholesterolemic subjects: a randomized crossover trial. Circulation 2004; 109: 1609-14.

[22] Cortés B, Núñez I, Cofán M, et al. Acute effects of high-fat meals enriched with walnuts or olive oil on postprandial endothelial function. J Am Coll Cardiol 2006; 48: 1666-71.

[23] Sabate J, Ang Y. Nuts and health outcome: new epidemiologic evedence. Am J Clin Nutr 2009; 89: 1643S-8S.

[24] Jiménez-Gómez Y, López-Miranda J, Blanco-Colio LM, et al. Olive oil and walnut breakfasts reduce the postprandial inflammatory response in mononuclear cells compared with a butter breakfast in healthy men. Atherosclerosis 2009; 204(2): e70-6.

[25] Esposito K, Marfella R, Ciotola M, et al. Effect of a Mediterranean-style diet on endothelial dysfunction and markers of vascular inflammation in the metabolic syndrome: a randomized trial. JAMA 2004; 292: 1440-6.

[26] Singh RB, Rastogi SS, Verma R, Bolaki L, Singh R, Ghosh S. An Indian experiment with nutritional modulation in acute myocardial infarction. Am J Cardiol 1992; 69: 879-85.
[27] Singh RB, Rastogi SS, Verma R, et al. Randomized, controlled trial of cardioprotective diet in patients with acute myocardial infarction: results of one year follow up. BMJ 1992; 304: 1015-9.

[28] Singh RB, Niaz MA, Sharma JP, Kumar R, Rastogi V, Moshiri M. Randomized, double blind,placebo controlled trial of fish oil and mustard oil in patients with suspected acute myocardial infarction:the Indian Experiment of Infarct Survival-4. Cardiovasc Durg Ther 1997; 11: 485-91.

[29] Singh RB, Pella D,DeMeester F. What to eat and chew in acute myocardial infarction. Eur Heart J 2006; 27: 1628-9.

[30] de Lorgeril M, Renaud S, Mamelle N, et al. Mediterranean alphalinolenic acid-rich diet in secondary prevention of coronary heart disease. Lancet 1994; 343(8911): 1454-9. Erratum in: Lancet 1995; 345 (8951): 738

[31] Renaud S, de Lorgeril M, Delaye J, et al. Cretan Mediterranean diet for prevention of coronary heart disease. Am J Clin Nutr 1995; 61(6 Suppl): 1360S-7S.

[32] Mechirova V, Singh RB, De Meester F, et al. Can wild whole seeds protect against cardiovascular disease. World Heart J 2008; 1: 137-8.

[33] Singh RB, Rastogi V, Niaz MA, Ghosh S, Singh R , Gupta S. Effect of fat modified and fruit and vegetable enriched diet on blood lipids in the Indian diet heart study. Am J Cardiol 1992; 70: 869-74.

[34] Singh RB, Rastogi SS, Niaz MA, Ghosh S, Singh R. Effects of fat modified and fruits vegetable enriched diets on blood lipids in the Indian diet heart study. Am J Cardiol 1992; 69: 869-74.

[35] Singh RB, Dubnov G, Niaz MA, et al. Effect of an IndoMediterranean diet on progression of coronary disease in high risk patients:a randomized single blind trial. Lancet 2002; 360: $1455-61$.

[36] Cicero AF, Borghi C, Derosa G, Di Gregori V, Bove M, Gaddi AV. Omega 3 polyunsaturated fatty acids supplementation and blood pressure levels in hypertriglyceridemic patients with untreated normal-high blood pressure and with or without metabolic syndrome: a retrospective study. Clin Exp Hypertens 2010; 32(2): $137-44$.

[37] De Meester F. Progress in lipid nutrition: the Columbus Concept addressing chronic diseases. In: Simopoulos AP, De Meester F, Eds. A balanced omega-6/omega-3 fatty acid ratio, cholesterol and coronary heart disease. World Review of Nutrition and Dietetics, (Simopoulos AP, series Ed). Basel: Karger; 2009; vol. 100: pp. 110-121.

[38] Li SC, Chen CY. Eating almonds may benefit subjects with type 2 and hyperlipidemia. Metabolism 2010; [Epub ahead of print]. 